\title{
A bi-objective model for the integrated frequency-timetabling problem
}

\author{
P. Ávila ${ }^{1}$, F. Irarragorri ${ }^{1} \&$ R. Caballero ${ }^{2}$ \\ ${ }^{1}$ Universidad Autónoma de Nuevo León, Mexico \\ ${ }^{2}$ Universidad de Malaga, Spain
}

\begin{abstract}
The urban transport planning process has four basic activities: network design, timetable construction, vehicle scheduling and crew scheduling. In this work we focus on the urban bus timetable construction problem which has two sub-activities: bus frequency calculation and bus departures setting. Typically, these sub-activities are done sequentially, which has some drawbacks: to overcome these drawback we propose to integrate both these sub-activities. We take into consideration: multiple planning periods, synchronizations events (a synchronization occurs when two buses from different routes arrive to a node within a time window), fixed and variable operations costs and uncertain demand in each period.
\end{abstract}

Keywords: frequency, timetable, integrated, fuzzy logic, multiperiod.

\section{Introduction}

In this paper is addressed the problem of urban transport planning . This process have four basic activities: network design, timetabling constructing, vehicle scheduling, and crew scheduling [1]. In this work we focus on the urban bus timetable construction problem which has two sub-activities: bus frequency calculation and bus departures setting. Typically, these sub-activities are done sequentially, which has some drawbacks, to overcome theme we propose to integrate both sub-activities mentioned above. The minimum frequency problem, is a fundamental problem for the schedulers because at least they have two objectives in conflict: the companies wish to minimize the operation costs and the passengers desire a minimum travel time. Timetable construction is a very 
important activity because it is the base of the success for the next activities of the public transport process as noted by Borndorfer et al. [2].

The minimum frequencies calculation and the timetable construction are two activities that schedulers face day to day. If the timetable is not planned correctly the schedulers used to modify it based on their experience and this can cause implications for the vehicle scheduling and crew scheduling activities and any change made in the minimum frequency and its timetable can cause to need a greater number of vehicles and drivers. In general, the timetables are important for a company because they help to maintain under control salaries, and others aspects of the operation, and the timetables are the base for a controlled and orderly operation.

In this paper we are tackling the bus timetable construction problem of an urban bus transport network, which is divided in two sub-activities: first for each scenario (covering a concrete planning period) bus frequencies are calculated for each route in the network, then bus departures are settled for each route in the network, were previously calculated frequencies are employed as a lower bound for the total departures programmed in each route in each scenario. The main scientific contribution of this work is the development of an integrated bi-objective mathematical model considering uncertainty in passengers demand to construct multi-period urban bus timetables.

We found papers considering the stochastic or fuzzy behaviour in some factors of bus planning and combining two phases of the urban transport process. For example, Berger et al. [3] propose a stochastic model for delay propagation and forecasts of arrival and departure events which is applicable to all kind of public transport.

Shangyao et al. [4] say most of the past research has used the average passenger demand as input to produce the final timetable and schedule, neglecting the variation of daily passenger demands in actual operations. So, they developed a stochastic-demand scheduling model and they employed simulation techniques. In order to show the behaviour of traveling choice under the influence of the cost, time and user preference, Zhua et al. [5] developed a variational inequality model to describe the stochastic multi-user and multi-modal mixed traffic equilibrium in their model, the mutual influences among different traffic modes are considered in a general traffic network. Hadas and Shnaiderman [6] developed an optimal model for public-transport frequency setting taking into account the costs associated with running empty-seats and passenger overload, and considering stochastic demand and travel time. Zhou et al. [7] establish a new stochastic nonlinear programming model to the driving timetable. They consider both interest of passengers and bus companies using stochastic analysis theory, programming theory.

In Section 2 we describe the problem we tackle, we give a brief introduction to fuzzy linear programming in Section 3, then in Section 4 we present the mathematical model we implemented, we presented the results we got in Section 5 and finally, in Section 6 we give the conclusions and future work. 


\section{Problem description}

In this paper, basically we want to determine the quantity of travels of each bus route and the departure time of each travel, in other words the frequencies and the timetable but not in a sequential way, how it had been executed traditionally, but in an integrated way. We are considering for this problem two objectives like, the cost (fixed and variable), which are to be minimized by the urban transportation enterprise and the other objective is to maximize synchronizations, which are a proxy measure for achieving overall lower passengers travel times. Synchronizations event are desired to occur in two situations: one is to avoid bus bunching and the other is to maximize passenger transfer and reduce passengers transfer times. to avoid bus bunching successive arrival at a node should be spaced by a minimum time, while minimization transfer times and maximization of synchronizations occur at synchronization nodes when two buses of two different routes are arriving to the node within a window time. We are also considering uncertainty in the passenger demand, where only lower and upper bounds are known. Also smooth transitions between adjacent periods with different demand are desired.

\section{Fuzzy linear programming}

Linear programming is one of the most frequently applied operations research technique. In the conventional approach value of the parameters of linear programming models must be well defined and precise. However, in real world environment, this is not a realistic assumption. In the real life problems there may exists uncertainty about the parameters. In such a situation the parameters of linear programming problems may be represented as fuzzy numbers [8]. Any linear programming model representing real world situations involves a lot of parameters whose values are assigned by experts. However, both experts and decision maker frequently do not precisely know the value of those parameters. Therefore it is useful to consider the knowledge of experts about the parameters as fuzzy data [9].

We consider, two kinds of fuzzy models: a complete fuzzy model where all parameters are fuzzy and a partial fuzzy model where some of the parameters are fuzzy, we can have a fuzzy objective function or a restriction or just an element in the restriction can be fuzzy. A fuzzy number A is a fuzzy set on the real line that satisfies the conditions of normality and convexity. In fact, any fuzzy number is defined by its corresponding membership function [8]. In this paper, we assume triangular fuzzy numbers which, in general, will be denoted by $\tilde{a}=(\underline{a}, a, \bar{a})$ where $\underline{a}$ and $\bar{a}$ denote the lower and upper limits of the support of a fuzzy number $\tilde{a} \in \mathrm{N}(\mathrm{R})$ with mode $a[10]$.

The problem of ranking fuzzy numbers has been extensively studied in the literature and a large collection of methods has been developed to solve it. As is well known, there are two main approaches to ranking fuzzy numbers, according either to (a) the use of a ranking function (index) or (b) by using methods assuming that the decision maker a priori chooses a degree of conformity for which the 
inequality may be considered as true by himself [10]. The method we employ in this work is k-preference index $F_{k}$, suggested for ranking fuzzy numbers. For a given level $k \in[0,1]$ if triangular membership functions are considered, if $\tilde{a} \leq k \tilde{b}$, then $k a+(1-k) \bar{a} \leq k b+(1-k) \bar{b}$ and $k a+(1-k) \underline{a} \leq k b+(1-k) \underline{b}$ [10]. We decided to use this method because its simplicity of implementation when we consider preferences of decision maker, besides, this method have been used in fuzzy programming for solving similar problems that the one being addressed in this paper.

\section{Mathematical model}

\section{Sets}

$M$ : Set of routes, $K$ : Set of nodes, $V$ : Set of periods, $B_{i j}^{v}$ : Set of pairs of nodes where potentially synchronize the routes $i$ and $j, J(i)$ : Set of routes which have common nodes with the route $i$.

\section{Variables}

$X_{i p}^{v}=1$ there is a trip in the route $i$ with departure time in the interval $p$ in the period $v$ y 0 otherwise.

$\alpha_{i p}^{v} \in\left(p \cdot H_{\text {min }_{i}}^{v}, p+1 \cdot H_{\text {min }_{i}}^{v}+g\right)$ iff $X_{i p}^{v}=1, \alpha_{i p}^{v}=0$ iff $X_{i p}^{v}=0$.

$Y_{i j k u p q}^{v}=1$ if the bus of the route $i$ with departure time in the interval $p$ and the bus of the route $j$ with departure time in the interval $q$ in the period $v$, arrive to the segment $k-u$ within the window time and 0 otherwise.

\section{Parameters}

$G^{v}$ : Number of trips in the period if we use a frequency equal to $H_{\max }^{v}$.

$P_{\max _{i}}^{v}$ : Maximum load of passengers in the route $i$ in the period $v$.

$P_{\text {maxd }}$ : Maximum load of passengers on board in the day in the route $i$.

$d_{i}^{v}$ : Desired occupancy of the bus in the route $i$ in the period $v$.

$P a s_{i}^{v}$ : Total passengers $/ \mathrm{km}$ in the route $i$ in the period $v$.

$L_{i}$ : Length of the route $i$.

$c a p_{i}^{v}$ : Bus capacity of the route $i$ in the period $v$.

$l_{k}$ : Length of the segment $k$.

$\beta_{i}^{v}$ : Percentage allowed of the route $i$ of exceed the load in the period $v$.

$H_{\text {max }_{i}}^{v}$ : Minimum headway of the route $i$ in the period $v$.

$H_{\text {min }_{i}}^{v}$ : Maximum headway of the route $i$ in the period $v$.

$T^{v}$ : Planning period; $\left[T_{i n i}^{v}, T_{\text {fin }}^{v}\right]$.

$T_{i n i}^{v}$ : Beginning time of the planning period $v$.

$T_{f i n}^{v}$ : Ending time of the planning period $v$.

$\gamma_{i}^{v}$ : Desired time before the end of the period $T^{v}$ for the last departure of the route $i$ in the period $v$.

$W_{\max _{i}}^{v}:$ Maximum window time for the route $i$ in the period $v$.

$W_{\min _{i}}^{v}$ : Minimum window time for the route $i$ in the period $v$.

$t_{i k}^{v}$ : Travel time from the origin point of the route $i$ to the segment $k$ in the period $v$. 
$\delta_{i j k u}^{v}$ : Minimum time the passenger needs to change from segment $k$ of the route $i$ to the segment $u$ of the route $j$ in the period $v$.

$\bar{P}_{\max _{i}}^{v}$ : Maximum load average of passengers on board of route $i$ in the period $v$.

$M C^{v}$ : Method applied to determine the frequency in the period $v$.

$f_{m r_{i}}^{v}$ : Minimum frequency required to satisfy the demand of the route $i$ in the period $v f_{m r_{i}}^{v}=\frac{\bar{P}_{\max _{i}}^{v}}{d_{i}^{v}}$.

FixedCost ${ }_{i}^{v}$ : Fixed cost for the route $i$ in the period $v$.

VariableCost ${ }_{i}^{v}$ : Variable cost for the route $i$ in the period $v$.

$P_{k}^{v}$ : Average of passengers on board in the segment $k$ in the period $v$.

$s_{j k}^{v}$ : Holding time of the route $j$ in the segment $k$ during the period $v$.

$t c_{i}^{v}=\left\lceil\frac{H_{\text {max }_{i}}^{v}}{H_{\text {min }_{i}}^{v}}\right\rceil, t f_{i}^{v}=\left\lfloor\frac{H_{\text {max }_{i}}^{v}}{H_{\text {min }_{i}}^{v}}\right\rfloor, t m_{i}^{v}=\left\lceil\frac{-T_{i n i}^{v}+\left(T_{f i n}^{v}-\gamma_{i}^{v}\right)}{H_{\text {min }_{i}}^{v}}\right\rceil$

$m c p_{i}^{v}=\max \left\{p-t c_{i}^{v}, N^{v}\right\}, m f p_{i}^{v}=\max \left\{p-t f_{i}^{v}, N^{v}\right\}, m s p_{i}^{v}=\max \{p-1,1\}$, $m u_{i}^{v}=\min \left\{t m_{i}^{v}+1, N^{v}\right\}$

$\overline{P_{k}^{v}}$ : Superior variance of the average of passengers on board in the segment $k$ in the period $v$.

$P_{k}^{v}$ : Inferior variance of the average of passengers on board in the segment $k$ in $\overline{\text { the }}$ period $v$.

$s_{j k}^{v}$ : Time (minutes) a bus of the route $j$ in the segment $k$ stays in the period $v$.

$\sigma$ : Confidence level $[0,1], h:$ Accomplish grade. $[\sigma, 1]$

$$
\begin{aligned}
& t c_{i}^{v}=\left\lceil\frac{H_{\text {max }_{i}}^{v}}{H_{\text {mini }_{i}}^{v}}\right\rceil, t f_{i}^{v}=\left\lfloor\frac{H_{\text {max }_{i}}^{v}}{H_{\text {min }_{i}}^{v}}\right\rfloor, t m_{i}^{v}=\left\lceil\frac{-T_{i i_{i}}^{v}+\left(T_{f i n}^{v}-\gamma_{i}^{v}\right)}{H_{\text {min }_{i}}^{v}}\right\rceil \\
& m c p_{i}^{v}=\max \left\{p-t c_{i}^{v}, N^{v}\right\}, m f p_{i}^{v}=\max \left\{p-t f_{i}^{v}, N^{v}\right\} \\
& m s p_{i}^{v}=\max \{p-1,1\}, m u_{i}^{v}=\min \left\{t m_{i}^{v}+1, N^{v}\right\} \\
& \text { FreMin }{ }_{i}^{v}=\min \left\{N^{v}, \max \left\{\left|G^{v}\right|, \sum_{p \in N^{v}} X_{i p}^{v}, f_{m r_{i}}^{v}\right\}\right\} \\
& \underline{\operatorname{FreMin}_{i}^{v}}=\min \left\{N^{v}, \max \left\{\left|G^{v}\right|, \sum_{p \in N^{v}} X_{i p}^{v}, \underline{f_{m r_{i}}^{v}}\right\}\right\} \\
& \overline{\operatorname{FreMin}_{i}^{v}}=\min \left\{N^{v}, \max \left\{\left|G^{v}\right|, \sum_{p \in N^{v}} \overline{X_{i p}^{v}}, \overline{f_{m r_{i}}^{v}}\right\}\right\}
\end{aligned}
$$

$$
\min \sum_{i \in M} \sum_{v \in V}\left(\text { FixedCost }_{i}^{v}+\text { VariableCost }_{i}^{v} \cdot L_{i} \cdot \sum_{p \in N^{v}} X_{i p}^{v}\right)
$$

$$
\max \sum_{i \in M} \sum_{j \in J(i)} \sum_{(k, u) \in B_{i j}^{v}} \sum_{v \in V} \sum_{p \in N^{v}} \sum_{q \in N^{v}} Y_{i j k u p q}^{v}
$$

s.t.

$$
\begin{array}{r}
M C 1=\frac{P_{\operatorname{maxd}_{i}}}{d_{i}^{v}} \\
M C 1 S=\frac{d_{i}^{v} \cdot \overline{\operatorname{Pmaxd}_{i}}}{\left(d_{i}^{v}\right)^{2}} M C 1 I=\frac{d_{i}^{v} \cdot \frac{\operatorname{Pmax}_{i}}{\left(d_{i}^{v}\right)^{2}}}{}
\end{array}
$$




$$
\begin{aligned}
& M C 2=\frac{P_{\max _{i}}^{v}}{d_{i}^{v}} \\
& M C 2 S=\frac{d_{i}^{v} \cdot \overline{P_{\max _{i}}^{v}}}{\left(d_{i}^{v}\right)^{2}} M C 2 I=\frac{d_{i}^{v} \cdot \underline{P_{\max _{i}}^{v}}}{\left(d_{i}^{v}\right)^{2}} \\
& M 3=\max \left(\frac{\operatorname{Pas}_{i}^{v}}{d_{i}^{v} \cdot L_{i}}, \frac{P_{\max _{i}}^{v}}{c a p_{i}^{v}}\right) M 3 S=\max \left(\frac{\overline{\operatorname{Pas}_{i}^{v}} \cdot d_{i}^{v} \cdot L_{i}}{\left(d_{i}^{v} \cdot L_{i}\right)^{2}}, \frac{\overline{P_{\max _{i}}^{v}} \cdot \operatorname{cap}_{i}^{v}}{\left(c a p_{i}^{v}\right)^{2}}\right) \\
& M 3 I=\max \left(\frac{P a s_{i}^{v} \cdot d_{i}^{v} \cdot L_{i}}{\overline{\left(d_{i}^{v} \cdot L_{i}\right)^{2}}}, \frac{P_{\max _{i}}^{v} \cdot c a p_{i}^{v}}{\left(\operatorname{cap}_{i}^{v}\right)^{2}}\right)
\end{aligned}
$$

$$
\begin{aligned}
M 4=\max \left(\frac{D e m_{i}^{v}}{d_{i}^{v}}, \frac{P_{\max _{i}}^{v}}{c a p_{i}^{v}}\right) M 4 S & =\max \left(\frac{\overline{D e m_{i}^{v}} \cdot d_{i}^{v}}{\left(d_{i}^{v} \cdot L_{i}\right)^{2}}, \frac{\overline{P_{\max _{i}}^{v}} \cdot c a p_{i}^{v}}{\left(c a p_{i}^{v}\right)^{2}}\right) \\
M 4 I & =\max \left(\frac{\overline{D e m_{i}^{v}} \cdot d_{i}^{v}}{\overline{\left(d_{i}^{v} \cdot L_{i}\right)^{2}}}, \frac{P_{\max _{i}}^{v} \cdot c a p_{i}^{v}}{\left(c a p_{i}^{v}\right)^{2}}\right)
\end{aligned}
$$

$$
\begin{array}{r}
\alpha_{i p}^{v} \leq X_{i p}^{v} \cdot\left(T_{i n i}^{v}+H_{\text {mini }_{i}}^{v} \cdot p\right) \\
\alpha_{i p}^{v} \geq X_{i p}^{v} \cdot\left(T_{i n i}^{v}+(p-1) \cdot H_{\text {mini }_{i}}^{v}+1\right) \forall v \in V, \forall i \in M, \forall p \in N^{v}
\end{array}
$$

$$
\begin{array}{r}
\sum_{p \in N^{v}} X_{i p}^{v} \geq(1-\text { GradoConf }) \cdot \overline{\text { FreMin }_{i}^{v}}+\text { GradoConf } \cdot \text { FreMin }_{i}^{v} \\
\sum_{p \in N^{v}} X_{i p}^{v} \geq(1-\text { GradoConf }) \cdot \underline{\text { FreMin }_{i}^{v}}+\operatorname{GradoConf} \cdot \text { FreMin }_{i}^{v} \\
v \in V ; i \in M
\end{array}
$$

$$
\begin{aligned}
\alpha_{i\left(t c_{i}^{v}\right)}^{v} \leq & \sum_{c=1}^{t f_{i}^{v}} X_{i c}^{v} \cdot M+\left(T_{i n i}^{v}+H_{\text {max }_{i}}^{v}\right) \\
& \sum_{c=1}^{\left(t c_{i}^{v}\right)} X_{i c}^{v} \geq 1 \forall v \in V, \forall i \in M
\end{aligned}
$$




$$
\begin{aligned}
& \alpha_{i p}^{v}-\alpha_{i,\left(m c p_{i}^{v}\right)}^{v} \leq T_{f i n}^{v} \cdot \sum_{c=m f p_{i}^{v}}^{m s p_{i}^{v}} X_{i c}^{v}+X_{i\left(m c p_{i}^{v}\right)}^{v} \cdot H_{\max _{i}}^{v} \\
& \alpha_{i p}^{v}-\alpha_{i\left(m s p_{i}^{v}\right)}^{v} \leq T_{f i n}^{v} \cdot\left(1-X_{i,\left(m s p_{i}^{v}\right)}^{v}\right)+X_{i p}^{v} \cdot H_{\text {max }_{i}}^{v} \\
& -\left(T_{f i n}^{v}\right) \cdot\left(1-X_{i,\left(m s p_{i}^{v}\right)}^{v}\right)-T_{f i n}^{v} \cdot\left(1-X_{i p}^{v}\right) \leq \alpha_{i p}^{v}-\alpha_{i,\left(m s p_{i}^{v}\right)}^{v}-H_{m i n_{i}}^{v} \cdot X_{i p}^{v} \\
& \sum_{c=m c p_{i}^{v}}^{m s p_{i}^{v}} X_{i c}^{v} \geq 1-\left(1-X_{i p}^{v}\right) \cdot\left(N^{v}+1\right) \\
& \forall v \in V \forall i \in M \\
& \alpha_{i\left(t m_{i}^{v}\right)}^{v} \geq-M \cdot \sum_{c=m u_{i}^{v}}^{N^{v}} X_{i c}^{v}+\left(T_{f i n}^{v}-\gamma_{i}^{v}\right) \\
& \sum_{c=t m_{i}^{v}}^{N^{v}} X_{i c}^{v} \geq 1 \forall v \in V, \forall i \in M, \forall p, l \in N^{v} \\
& -1 \cdot\left(\alpha_{i p}^{v}+t_{i k}^{v}+\delta_{i j k}^{v}\right)+\left(\alpha_{j q}^{v^{*}}+t_{j u}^{v}+s_{j k}^{v}\right) \geq W_{\min _{i}}^{v}-M \cdot\left(1-Y_{i j k u p q}^{v}\right) \\
& -1 \cdot\left(\alpha_{i p}^{v}+t_{i k}^{v}+\delta_{i j k}^{v}\right)+\left(\alpha_{j q}^{v^{*}}+t_{j u}^{v}+s_{j k}^{v}\right) \leq W_{\max _{i}}^{v}+M \cdot\left(1-Y_{i j k u p q}^{v}\right) \\
& \alpha_{i p}^{v}+\alpha_{j q}^{v} \geq 2 \cdot Y_{i j k u p q}^{v} ; v, v^{*} \in V ; i \in M ;(k, u) \in B_{i j}^{v} \text {; } \\
& j \in J(i) ; p \in N^{v}, q \in N^{v^{*}}, v^{*} \leq v
\end{aligned}
$$

The model consists of 2 objective functions, the first objective function (1) minimize the total cost. We are considering a fixed and a variable cost which it is affected by the length of the route and the quantity of departures made by the route in the period. The second function (2) is to maximize the number of synchronizations between two bus routes. These objective functions are subjected to constraints of frequency (3-6) which indicate the minimum quantity of units that a route needs to satisfy the demand. With constraint (7) it is ensured that if there is not a departure in the period $v$ for the route $i$ in the segment $p$ then it is not assigned a valid departure time to the interval $\alpha_{i p}^{v}$. In constraint (8) the quantity of departures must be the maximum between the number of departures determined by the maximum headway, the minimum frequency and the frequency got it whit a method, which satisfies the maximum load point, in this way we are warranting accomplish of the demand. Demand is a fuzzy number, so we implemented a ranking method to compare these numbers. Constraint (9) indicates that the first departure time must be less or equal to the maximum headway. Constraint (10) is for the consecutive departures, here indicates that the departure time must be 
between the minimum and maximum headway. For the last departure (11) we say the departure time must be between the end of the period and desired time. The constraint (12) represents the synchronization which indicates when two bus of different routes arrives to a synchronization node between a window time and taking into account the transfer times, the permanence time in a node and the travel time, then there is a synchronization.

\section{Results}

First, we wanted to know how the level of demand influence which frequency method should be used, so we generated 70 small instances varying the demand between periods. The instance presented here has just 1 route, 3 periods and 15 segments. In this example we can see that when demand is almost the same in a period, the method we use is the number 1 , when we have a low demand and it varies inside the period we use method number 4 , and when demand change but not significantly the method we employ is number 2 . Very similar behaviour was noticed in all other instances.

Table 1: Demands of periods.

\begin{tabular}{|c|c|c|}
\hline Periods & Demand & Method \\
\hline Period 1 & 203208205215213 & 1 \\
\hline Period 2 & 3305508 & $3-4$ \\
\hline Period 3 & 103270100190290 & 2 \\
\hline
\end{tabular}

Then we use those same instances to discover the influence of the uncertainty of demand on the objective values, in this experiment we used a weighted sum method of the two objectives with $0,0.25,0.50,0.75$ and 1 as weights and we use these values also for the confidence levels. As expected objective values changed when varying confidence levels, the cost obtained with the confidence level 0 was higher than the cost obtained with confidence level 1 , while the synchronizations do not change significantly because they are time dependent and we consider time as deterministic, these results can be observed in Table 2 .

We then conducted another experiment where we tested our model using 25 instances that were randomly generated; those instances are divided into small, medium and large according to the synchronization density. While the number of routes, number of periods and segments were kept almost constant, because we were interested to investigate how synchronization density affects the solution. The density of synchronization was generated as a percentage of the combination of routes and segments, those percentages were $1 \%-2 \%, 2 \%-4 \%$ and $4 \%-7 \%$ for small, medium and large respectively. 
Table 2: Fuzzy results.

\begin{tabular}{|c|c|c|c|c|}
\hline Weights & Confidence & Cost & Synchr. & Departs \\
\hline $.5-.5$ & 0 & 9714050.12 & 44 & 21 \\
\hline $.5-.5$ & 0.25 & 9231890.12 & 42 & 20 \\
\hline $.5-.5$ & 0.5 & 8267570.12 & 36 & 18 \\
\hline $.5-.5$ & 0.75 & 7303250.12 & 30 & 16 \\
\hline $.5-.5$ & 1 & 6338930.12 & 24 & 14 \\
\hline $.75-.25$ & 0 & 9290690.12 & 40 & 20 \\
\hline $.75-.25$ & 0.25 & 8326370.12 & 34 & 18 \\
\hline $.75-.25$ & 0.5 & 7362050.12 & 28 & 16 \\
\hline $.75-.25$ & 0.75 & 6397730.12 & 22 & 14 \\
\hline $.75-.25$ & 1 & 5433410.12 & 16 & 12 \\
\hline
\end{tabular}

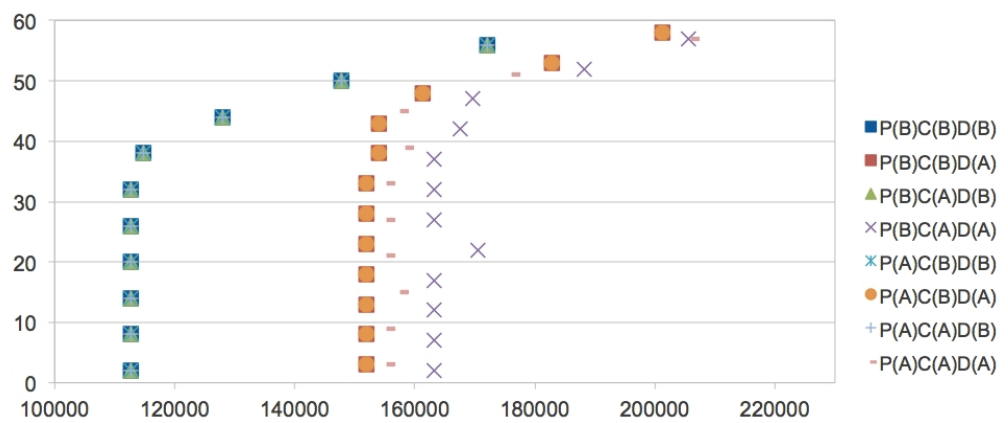

Figure 1: Front compound by weak non dominated and non dominated points.

For the experiment we consider three factors: level of preference $(0.25$ and 1$)$, level of confidence (01. and 0.9) and level of demand (0-low and 1-high) the levels for preference (to what extend the decision maker allows the fuzzy constraints to be violated) are 0.25 and 1, two levels of confidence (degree of optimism of the decision maker) 0.1 and 0.9. and two levels for uncertainty in demand 0.60 lower and 1.35 higher. By combining the factors levels we got 8 different combinations and then have generated 10 solutions points for constructing the Pareto front for each combination. When solved the instances, all of them behaved almost in the same way, the gap we got was around $10 \%$ in a reasonable time. In Figure 1 you can find a graphical representation of the generated fronts by the combination of levels of the three factors: Preference (P), Confidence (C) and Level of Uncertainty in Demand (D), and their levels: High (A), Low (B). So a legend CP(A)(B)D(B) can be read as High Preference, Low Confidence and Low Uncertainty in Demand. As can be easily seen the best results are achieved for the combination of level of 
factors where the Uncertainty in Demand is at its lower level independently of the level of the remaining two factors. But when the Uncertainty in Demand is at its higher level, best results are achieved when Confidence is at is lower level. The worst results were achieved when the Uncertainty in Demand is at its higher level and also Confidence is at its higher level. These results agree with common sense. We got similar results with all other instances.

\section{Conclusions}

In this paper we proposed a partial fuzzy mathematical model for the integrated frequency and timetabling problem with uncertain demand with two objectives.

We show how the behavior of demand affects the selection of a frequency method for calculating minimal frequencies. Finally, we showed how varying different factors like preference, confidence and uncertainty in demand influence in the solutions obtained. Then, all this factors depend on the decision maker experience and preferences. In all cases we got a gap around $10 \%$ in a reasonable time and the behavior of the solutions was very similar. Better results are obtained with a good estimation of demand. Here we used only one method to rank fuzzy number: k-preference, in the future we would like to implement another ranking methods. Also we would like to incorporate uncertainty of time. Besides, we would like to experiment with column generation, valid inequalities and decomposition techniques.

\section{References}

[1] Ceder, A., Public transit planning and operation: theory, modelling and practice. Elsevier: USA, 1st edition, 2007.

[2] R. Borndorfer, M. Grotschel, \& M.E. Pfetsch, Models for line planning in public transport. Computer-aided Systems in Public Transport, Springer Berlin Heidelberg, volume 600, pp. 363-378, 2008.

[3] Berger, A., Gebhardt, A., Muller-Hannemann, M. \& Ostrowski, M., Stochastic delay prediction in large train networks. 11th Workshop on Algorithmic Approaches for Transportation Modelling, Optimization, and Systems, ed. S.K. A. Caprara, Oasics: Germany, pp. 109-120, 2011.

[4] Y. Shangyao, C. Chi \& C. Tang, Inter-city bus rounting and timetable setting under stochastic demands. Transportation research part A, 40(1), pp. 572586, 2006.

[5] Zhu, C., Jia, B., Li, X. \& Gao, Z., A stochastic mixed traffic equilibrium assignment model considering user preferences. Procedia Social and Behavioral Sciences, 43(0), pp. 466 - 474, 2012.

[6] Y. Hadas \& M. Shnaiderman, Public-transit frequency setting using minimum-cost approach with stochastic demand and travel time. Transportation Research Part B: Methodological, 46(8), pp. 1068 - 1084, 2012. 
[7] L. Zhou, H. Hui \& B. Liu, Synthetically improved genetic algorithm in stochastic programming model in public traffic dispatch system. Machine Learning and Cybernetics (ICMLC), 2010 International Conference on, volume 3, pp. 1602-1606, 2010.

[8] N. Mahdavi-Amiri, S. Hadi Nasseri \& A. Yazdani, Fuzzy primal simplex algorithms for solving fuzzy linear programming problems. Iranian Journal of Operations Research, 1(2), pp. 68 - 84, 2009.

[9] A. Kumer, J. Kaur \& P. Singh, Solving fully fuzzy linear programming problems with inequality constraints. International Journal of Physical and Mathematical Sciences, 1(1), pp. 6 - 18, 2010.

[10] L. Campos \& J.L. Verdegay, Linearprogrammingproblems and ranking of fuzzy numbers. Fuzzy sets and systems, 1(32), pp. 1 - 11, 1989. 